\title{
HPV infection upregulates the expression of ZNT-1 in condyloma acuminatum
}

\author{
Li Niu, ${ }^{1}$ Xiaoying Chu, ${ }^{1}$ Yaofei Jiang, ${ }^{2,3}$ Wei Zeng ${ }^{2,3}$ \\ ${ }^{1}$ Department of Pathology, Zhongnan Hospital of Wuhan University, Wuhan \\ ${ }^{2}$ Hubei Cancer Clinical Study Centre and Hubei Key Laboratory of Tumor Biological Behaviors, Zhongnan Hospital, \\ Wuhan University, Wuhan \\ ${ }^{3}$ Department of Radiation and Medical Oncology, Zhongnan Hospital, Wuhan University, Wuhan, China
}

Condyloma acuminata (CA) are benign anogenital warts caused by human papillomavirus (HPV) infection with a high recurrence rate. Despite its high contagiousness, high recurrence rate and potential for malignant transformation, effective treatments for CA have not yet been developed. Accordingly, it is necessary to clarify the mechanisms underlying CA development. Zinc ( $\mathrm{Zn})$ is stably maintained in the weight of human body. Skin is the third most $\mathrm{Zn}$-abundant tissue in the body. $\mathrm{Zn}$ is present as a divalent ion $\left(\mathrm{Zn}^{2+}\right)$ in cells and does not need a redox reaction upon crossing the cellular membrane. Zn transporters (ZnTs; SLC30A) and Irt-like proteins (ZIPs; SLC39A) are involved in $\mathrm{Zn}^{2+}$ efflux and uptake, respectively. ZnT1 is one of the ZnTs, which associates with the development of HPV. However, the role of ZnT1 regulation in the CA caused by HPV infection remains unknown. A multigroup case-control study was designed to investigate the expression and significance of the ZnT1 in patients with CA infected with HPV and in normal vulva controls. ZnT1 was assessed by immunohistochemistry in 44 patients with CA at Zhongnan Hospital of Wuhan University 2019-2020. Samples were analyzed by paraffin embedding and sectioning and hematoxylin-eosin and immunohistochemical staining. Immunohistochemical methods detected specific, dark brown, positive staining of ZnT1 in the keratinocytes of epidermis. We verified that the expression levels of ZnT1 that interact with HPV were upregulated in the CA groups independently of genotype compared with the control group. And then we found that the HPV risk grade in CA patients has a certain correlation with ZnT1 expression. These findings showed that HPV infection upregulated the expression of ZnT1 in CA. Additionally, there were obvious differences in the expression of ZnT1 between the different HPV risk grade infection groups. The higher the HPV risk grade, the stronger the ZNT1 protein expression. This study provided new insights into the sign pathway to HPV infection.

Key words: Condyloma acuminata; Znic transporters 1; human papillomavirus; immunohistochemical staining.

Correspondence: Wei Zeng, Department of Radiation and Medical Oncology, Zhongnan Hospital of Wuhan University, Wuhan, Hubei, 430071, China. Tel. +86.027.67813043. E-mail: zengweixh@126.com

Contributions: LN, WZ, designed the experiments and wrote the manuscript; LN, performed the experiments; $\mathrm{XC}, \mathrm{YJ}$, collected the samples and analyzed the data. All the authors have read and approved the final version of the manuscript and agreed to be accountable for all aspects of the work.

Conflict of interest: The authors declare that they have no competing interests, and all authors confirm accuracy.

Availability of data and materials: The data used to support the findings of this study are available from the corresponding author upon request.

Ethical Approval: All procedures with patients in this study were conducted in accordance with the Human Ethics Committee of Zhongnan Hospital of Wuhan University, Hubei, China. This article does not contain any studies with animals.

Funding: This work was supported by National Natural Science Foundation of China (NO.81600108) and Zhongnan Hospital of Wuhan University Science, Technology and Innovation Seed Fund, Project (No. znpy 2019069). 


\section{Introduction}

Condyloma acuminatum (CA) is a sexually transmitted disease caused by human papilloma virus (HPV) infecting the perianal and genitourinary organs. In the past ten years, the incidence of CA has shown a linear upward trend among the sexually transmitted diseases in China. ${ }^{1}$ The probability of contracting HPV among sexually active persons is higher year by year. ${ }^{2}$ HPV virus belongs to DNA virus, and the squamous epithelium of human skin and mucous membrane is important host. ${ }^{3} \mathrm{HPV}$ infection has three manifestations: clinical infection (typical CA), subclinical human papillomavirus infection (SPI) and latent human papillomavirus infection (LPI). ${ }^{4}$ SPI is an indicator of unobvious skin lesions seen by naked eyes while the acetic acid white test is positive ${ }^{5}$ The pathological manifestations are squamous epithelial atypical hyperplasia, visible or suspicious hollow cells and the HPV test is positive. ${ }^{5}$ LPI is the appearance of local skin and mucous membranes and the acetic acid white test is negative, but the presence of HPV can be detected by molecular biology methods. ${ }^{6}$ Studies have shown that the occurrence and multiple recurrences of CA are related to the disorders of local cellular immunity, development, differentiation and cell growth of patients.

$\mathrm{Zn}^{2+}$ is one of the important factors in the enzymatic reaction and transcription process in human physiological activities. ${ }^{1,8} \mathrm{About} 10 \%$ of human proteins bind to $\mathrm{Zn}^{2+}$ and participate in physiological processes. ${ }^{8} \mathrm{Zn}^{2+}$ is mainly involved in the development, differentiation and growth of cells in skin tissues which is the largest organ in the human body. ${ }^{9} \mathrm{Zn}^{2+}$ depends on $\mathrm{Zn}$ transporters (ZnTs) efflux and uptake, and different cells expresses different ZnTs. ${ }^{10}$ Abnormal changes in ZnTs are associated with the occurrence and development of a variety of malignant tumors, which has been confirmed in the progression of breast cancer and pancreatic cancer. However, there are few reports on the ZnTs in CA. ZNT1 is one of the zinc ion transporters in skin tissue. Studies have shown that ZNT1 interacts with EVER1 or EVER2 proteins in epidermodysplasia verruciformis (EV) disease in the nuclear membrane and Golgi apparatus. ${ }^{11}$ Compared with healthy individuals, the concentration of free $\mathrm{Zn}$ in the nucleus of squamous epithelial cells of EV patients is increased, suggesting that the complex of ZnTI and EVERs regulates the transport of $\mathrm{Zn}^{2+}$ in the nucleus, potentially changing cell functions and increasing HPV replication and promoting the occurrence of diseases ${ }^{11}$.

CA is one of the typical diseases related to HPV infection. At present, most hospitals in China mainly adopt ThinPrep cytology test (TCT - exfoliative cytology) method to screen for HPV infection. In pathological diagnosis, the detection of p16 protein is mainly used to infer HPV infection. ${ }^{12}$ The diffuse expression of p16 protein and the high expression of $\mathrm{Ki}-67$ proliferation index in pathological diagnosis indicate HPV infection and squamous intraepithelial lesions (CIN in the usual sense). ${ }^{13}$ However, the application of p16 protein in HPV detection is not enough to accurately represent HPV infection. In clinical work, we need more sensitive and specific detection indicators to confirm HPV infection. This study confirmed the expression of ZNT1 in CA from another view and indirectly proved that the expression of ZNT1 is related to HPV.

\section{Materials and Methods}

\section{Data screening}

We collect 44 cases with CA at Zhongnan Hospital of Wuhan University from December 31, 2019 to December 31, 2020, excluding patients who have not undergone HPV testing, tumor patients, and other patients with incomplete clinical information. The 44 cases ranged in age from 20 to 59 years old, and the median age was 28 years.

\section{HPV-DNA specimen collection and detection}

The lesion was exposed and was performed routine disinfection. First, a sterile cotton swab soaked with normal saline was used to clean the lesion surface of the patient for special use. The exfoliated cell collector was wiped several times on the surface of normal foreskin, typical wart body and subclinical infected skin lesions. Expressed epithelial cells were collected (at least 3 times), placed in sterile collection tubes, and the samples were labeled for HPV-DNA detection. HPV type was detected by PCR-membrane hybridization. The laboratory of our hospital uses the HPV test kit approved by the China Food and Drug Administration. The exfoliated epithelial cell samples were added with the corresponding reagents according to the instructions to extract DNA. The results could be determined by the steps of amplification, hybridization and coloration after membrane washing. Distinguish $21 \mathrm{HPV}$ genotypes in the test specimens, including 13 high-risk HPV 16, $18,31,33,35,39,45,51,52,56,58,59,68$, and 5 medium-risk HPV 36, 53, 66, 73, 82 and 3 low-risk HPV types 6, 11, 81. At the same time, the biopsy tissues of patients who tested negative for HPV infection by HPV test kit were used as negative controls.

\section{Immunohistochemistry analysis}

The specimens were fixed in $4 \%$ neutral formalin, dehydrated and embedded in paraffin. Then, hematoxylin-eosin and immunohistochemistry were used to stain 3-4 $\mu \mathrm{m}$ sections, and Leica's envision method was used for analysis. According to the manufacturer's instructions, the antibodies in this study included ZnT1 (Proteintech Group, Rosemont; IL, USA; 1:200 dilution), p16 and Ki-67 (Proteintech Group; 1:200 dilution). After the sections were incubated with $0.02 \%$ Sigma-Aldrich (St. Louis, MO, USA) and $0.005 \%$ hydrogen peroxide in $0.05 \mathrm{M}$ Tris $\mathrm{HCl}$ buffer at room temperature for $10 \mathrm{~min}$, brown positive reaction appeared. Positive control was established in each part. Immunohistochemistry was performed according to the following criteria: the target proteins stained black and brown in the nucleus represent positive. However, the positive staining was not related to the intensity and distribution in the tissues.

\section{Statistical analysis}

The protein expression level was determined by densitometry of the bands using Image-pro Plus 6.0 analysis software. All statistical analyses were performed using SPSS15.0 software package. All values are expressed as mean $\pm \mathrm{SD}$. Statistical analysis of the data uses analysis of variance, followed by $t$-test. If $\mathrm{p}<0.05$, the difference is considered significant.

\section{Results}

\section{HPV-DNA testing in patients with CA}

In this study, HPV-DNA detection were positive in $44 \mathrm{CA}$ patients, which including 21 cases of high-risk type HPV infection (47.73\%), 8 cases $(18.18 \%)$ of medium risk type HPV infection; low risk type HPV infection in 15 cases (34.09\%) (Table 1).

\section{ZnT1 protein was diffusely and highly expressed in CA}

Microscopically, papillary tissue growth was observed in patients with CA. Immunohistochemical methods detected specific, dark brown, positive staining of p16, Ki-67 and ZnT1 in the 
squamous epithelium. p16, as a tumor suppressor gene, overexpressed indicates the abnormal proliferation of cells with persistent HPV infection. Ki-67 is a nuclear antigen gene that marks the cell cycle progression and proliferation, which overexpression indicates that the cells are in the proliferative phase of the cell cycle. In normal physiologically functioning cells, the expression of p16 and Ki-67 antagonizes each other and does not occur simultaneously. If both p16 and Ki-67 are overexpressed at the same time, it indicates the dysregulation of cell cycle. Therefore, the simultaneous detection of p16 and Ki-67 in the same cell can be used as a marker for cell cycle dysregulation, which is associated with the carcinogenic transformation induced by high-risk HPV viruses. In this study, we found the P16 strong and diffused positive expression in the squamous epithelium consisted with the expression of Ki-67 (Figure 1 A,B). ZnT1 positive cells are mainly found in the epidermis and are expressed throughout the squamous epithelium. The positive signal is located in the nucleus and (or) cytoplasm of the squamous epithelium, with brown or tan particles. High ZnT1 expression can be observed in the tissues of CA patients (Figure 1C). In the study, we also found that the expression of p16 and $\mathrm{Ki}-67$ has a certain correlation with the expression of ZnT1. That is to say, when the expression of p16 and $\mathrm{Ki}-67$ in CA patients is diffusely and strongly positive in the epidermis, the expression pattern of ZnT1 is the same (Figure 1).

\section{The expression of $\mathrm{ZnT} 1$ was correlated with HPV risk grading}

In the study, the expression of ZNT1 is divided into four levels, where "-" means negative; "+" means weak positive expression; "++" means strong positive expression; "+++" means diffuse strong positive expression.

Table 1. HPV-DNA testing in patients with CA.

$\begin{array}{lccc}\text { Age (years) } & \text { High risk (n) } & \text { Medium risk (n) } & \text { Low risk (n) } \\ \leq 30 & 12 & 4 & 8 \\ 31-50 & 6 & 4 & 5 \\ \geq 51 & 3 & 0 & 2\end{array}$

High risk type: HPV 16, 18, 31, 33, 35, 39, 45, 51, 52, 56, 58, 59, 68; medium risk type: HPV 36, 53, 66, 73,82 ; low risk type: $\operatorname{HPV} 6,11,81$.
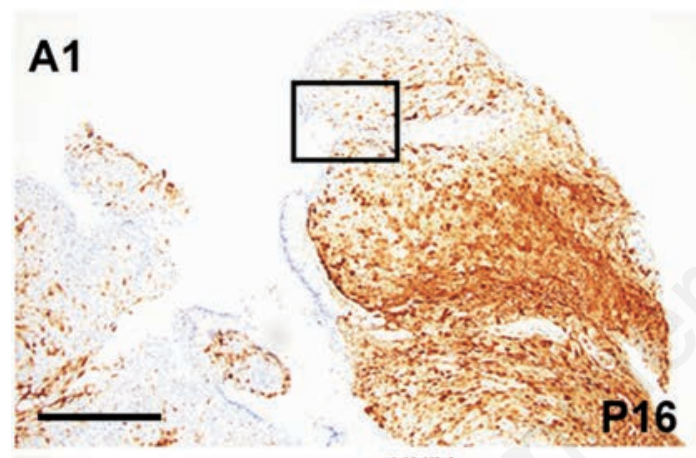

\section{B1}
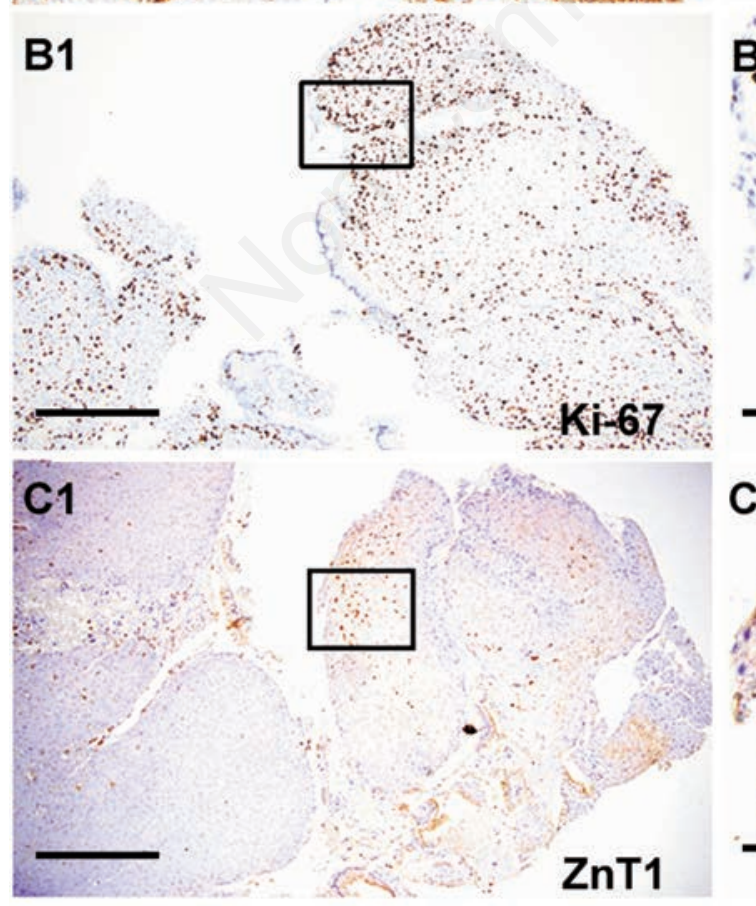

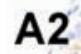

A2
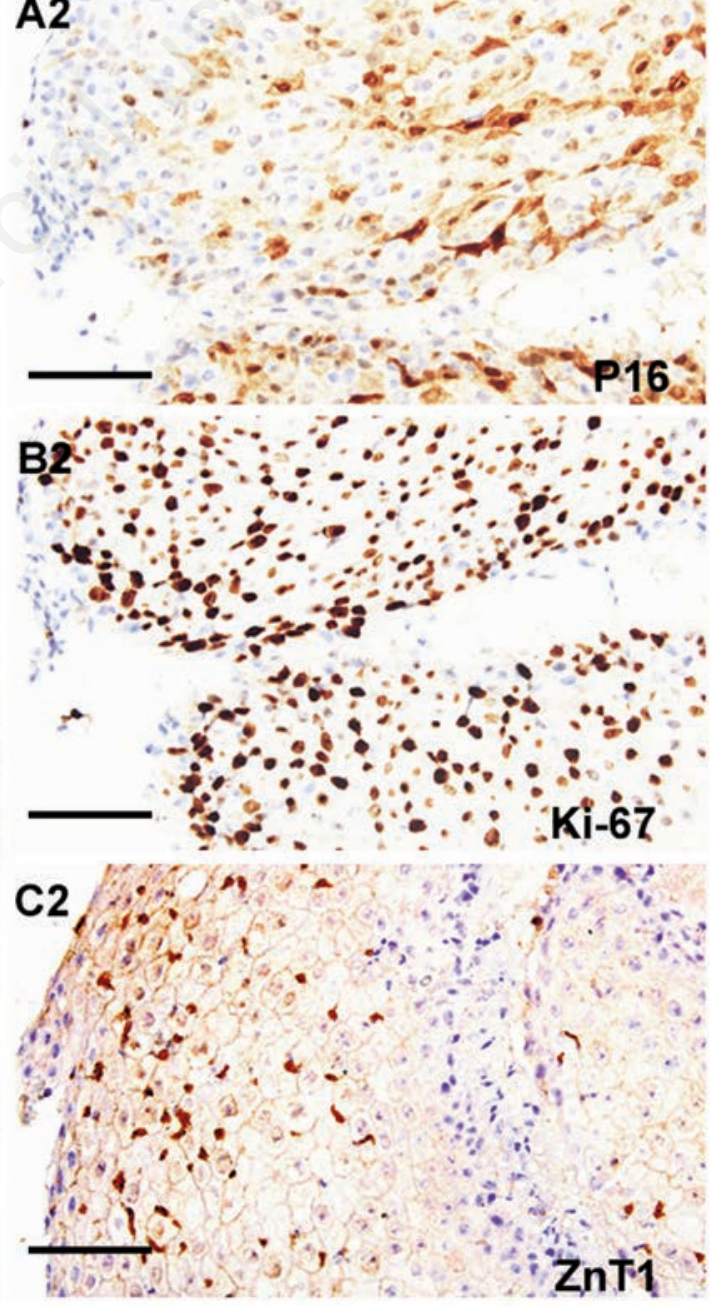

Figure 1. Immunohistochemical characteristics of $\mathrm{ZnT} 1$. The dark brown positive staining of p16, $\mathrm{Ki}-67$ and $\mathrm{ZnT} 1$ is seen in the $\mathrm{CA}$ patients. A,B,C, 40×; scale bar: $100 \mu \mathrm{m})$. A1, B1 and C1 are magnifications of A-C (100x); scale bar: $25 \mu \mathrm{m}$. 
The results showed that among 21 cases of HPV high-risk infection, 2 cases of ZNT1 weak expression accounted for $9.52 \%$ $(2 / 21)$; 4 cases of strong expression accounted for $19.05 \%(4 / 21)$; ZNT1 diffusely strongly positive expression cases accounted for the largest number. There are 14 cases accounting for $66.67 \%$ (14/21). Among all HPV high-risk infections, only one patient had negative expression of ZNT1 protein. Therefore, we found that the ZNT1 protein expression rate among HPV high-risk infections was as high as $95.24 \%(20 / 21)$ (Figure 2).

We found that in HPV-infected CA patients, the expression of $\mathrm{ZnT} 1$ is correlated with the risk grade of HPV. The expression of ZnT1 is weak in patients with low-risk HPV infection, and the expression of ZnT1 is stronger in patients with high-risk HPV infection. The results of the study showed that there were 21 patients with high-risk HPV infection, of which 14 patients showed diffuse and strong positive expression of ZNT1 protein, accounting for $31.82 \%$ (14/44). The expression of ZNT1 protein in patients with intermediate-risk HPV infection mainly showed weak positive and strong positive expression, accounting for $13.64 \%(6 / 44)$ of the total number of patients with intermediaterisk HPV infection. The expression of ZNT1 protein in patients with low-risk HPV infection is mainly negative and weakly positive, of which the negative ratio accounts for $11.36 \%(5 / 44)$, and the weakly positive expression accounts for $18.18 \%(8 / 44)$ (Table 2). While faint $\mathrm{ZnT} 1$ expression is seen in the normal control group (results not shown). The expression level of ZnT1 in the tissues of CA patients was significantly higher than that of the normal control group, and the difference was statistically significant $(\mathrm{p}<0.05)$. In the study, we found that $\mathrm{ZnT} 1$ expression is stronger in CA patients infected with HPV (high-risk type) (Table 2).

\section{Discussion}

HPV is a double-stranded DNA virus, and its main route of transmission is through sexual contact. ${ }^{3}$ Under special circumstances, it can also be transmitted through non-sexual contact, such as close contact, autologous vaccination, through the uterus or delivery from pregnant women infected with HPV vertical transmission to newborns. ${ }^{14}$ As the most widely spread sexually transmitted virus in the world, the lifetime infection rate of the population is about $75 \% .{ }^{15} \mathrm{HPV}$ infection is the most common sexually transmitted disease between the sexes. It is closely related to the onset of a variety of precancerous lesions and malignant tumors, causing a serious economic and social burden. ${ }^{16}$ Perianal and external genital skin and mucous membranes infected with HPV can be clinically manifested as dominant CA and subclinical and latent infections without obvious skin lesions. ${ }^{17}$ Epidemiological data indicate that most HPV infections do not show typical damage, and more often subclinical infections and latent infections are the main manifestations. ${ }^{18} \mathrm{HPV}$ can enter the skin through tiny damages or

Table 2. Expression strength of ZnT1 in CA tissues.

\begin{tabular}{lcccc} 
Group & \multicolumn{4}{c}{ Expression strength (grade) } \\
& - & + & ++ & +++ \\
High risk (n) & 1 & 2 & 4 & 14 \\
Medium risk (n) & 1 & 4 & 2 & 1 \\
\hline Low risk (n) & 5 & 8 & 1 & 1 \\
\hline
\end{tabular}
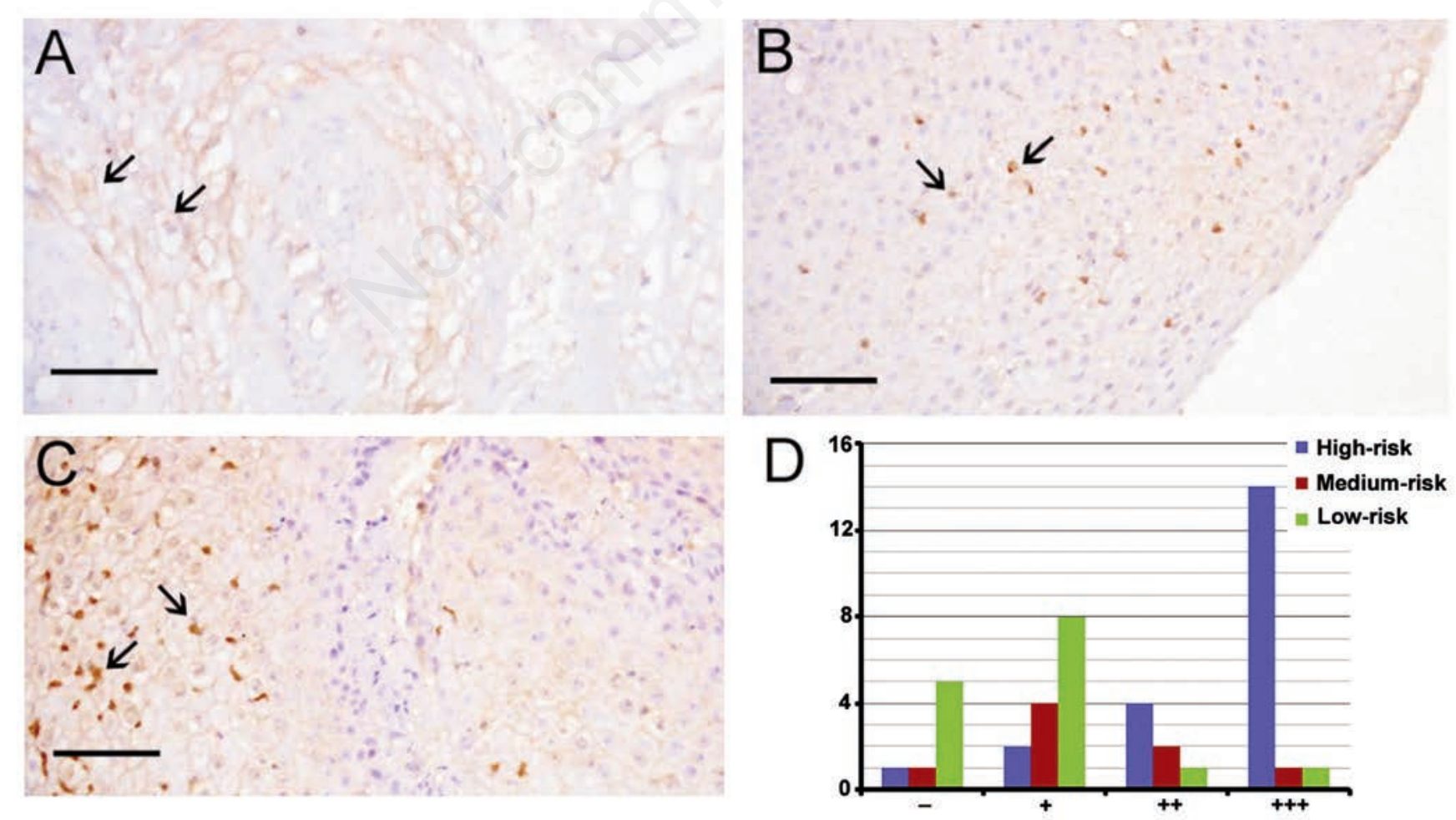

Figure 2. Expression strength of ZnT1 in high-risk CA cases. A) +; B) ++; C) +++. A-C (100×); scale bar: $25 \mu \mathrm{m}$. 
epithelial cells on the mucosal surface and infect the basal keratinocytes of the genitals and oral mucosa through further damage and micro damage. ${ }^{19}$ At present, more than $200 \mathrm{HPV}$ subtypes have been found, of which about 40 are related to the occurrence of anal and genital CA. ${ }^{20}$ These diseases that pose a serious threat to health often have no specific skin lesions in the early clinical stage, and most of the clinical manifestations are mainly itching in the affected area. ${ }^{21}$ Therefore, for patients with itchy perianal and external genital skin, timely HPV screening of skin exfoliated cells in the affected area has important clinical significance for the early prevention and treatment of atypical skin lesions, precancerous lesions, and cancerous changes caused by persistent HPV infection. ${ }^{22}$ In recent years, studies on HPV infection involved in the pathogenesis of CA are very limited. It mainly focuses on the role of high-risk carcinogenic HPV 16 and HPV 16 viruses in keratinocytes. ${ }^{23}$ In this study, we found that the expression of ZnT1 was elevated in HPV-infected CA patients. However, little is known about the interaction between HPV and ZnT1 currently. This leads to further speculation about the relationship between HPV and ZnT1.

In the research on the pathogenesis of skin diseases, the relationship between EV and HPV infection is mentioned. ${ }^{24}$ The article describes $\mathrm{EV}$, this rare lifelong autosomal recessive skin disease, which was recently classified as primary sexual immunodeficiency. ${ }^{25}$ It is related to ubiquitous $\beta$-HPV infection, but has nothing to do with other pathogens, including skin or genital $\alpha$-HPV, $\gamma$-HPV and other factors. ${ }^{26}$ Therefore, EV is considered as a potential model for the role of viruses in skin cancer. ${ }^{27}$ In the study of this model of EV, it was found that the mechanism of HPV infection is related to the mutation of EVER1 and EVER2 genes. ${ }^{11}$ Homozygous mutations of these two genes indicate that EV is susceptible to HPV. The discovery of EVER1 and EVER2 genes has opened up a new way to find the molecular background of the antiHPV barrier.

In addition, studies have found that both EVER1 and EVER2 interact with zinc transporter 1 (ZnT-1). ${ }^{11} \mathrm{ZnT}-1$ is a membrane protein responsible for zinc outflow and anti-zinc toxicity. It is reported in the literature that multiple interaction targets were found in EVER1, EVER2 and ZnT-1 in the yeast two-hybrid screening experiment. ${ }^{11}$ Both transmembrane virus E5/E8 and cellular EVER proteins interact with zinc transporter ZnT1 which may regulate zinc homeostasis. The topology of this network highly suggests that EVER protein and ZnT-1 exist as EVER1EVER2-ZnT-1 anchor complexes on the endoplasmic reticulum membrane. Studies reported that the ZnT-1-EVER complex play an important role in transport of free zinc inside keratinocytes in order to regulate the balance in its local concentration. This suggests that EVER2 regulates free zinc homeostasis and modulates its functions through ZnT-1 in human keratinocytes, especially in the transcriptional function of free zinc. ${ }^{28}$ Because of this, it has been reported in the literature that the ZnT-1-EVER complex proteins on specific signal transduction pathways leading to synthesis of transcription factors such as c-Jun N-terminal kinase (JNK) and phosphoinositide 3-kinase (PI3K), which belongs to the AP-1 family, and Elk-1. ${ }^{29}$ HPV16 E5 protein inhibits EVER and ZnT-1 activities which implicated that interaction between the HPV E5 and EVER- ZnT-1 complex might facilitate the concentration of free zinc needed for expression of viral genomes. These data suggest that inhibition of EVER-ZnT-1 complex by E5 protein (genital HPV) or mutation (EV HPV) is crucial for the HPV life cycle in keratinocytes. On this basis, the expression of ZnT1 and EVERZnT-1 complex can also affect the homeostasis of free zinc, resulting in a vicious cycle. Therefore, we speculate that ZNT1 protein is also involved in the pathogenesis of HPV infection. Our study found that ZNT1 protein is expressed in CA patients with HPV expression. There is a certain correlation between the intensity of ZNT1 protein expression and the risk grade of HPV. The expression of ZNT1 in the HPV high-risk group is diffusely strong. The results found in these studies also just confirmed our previous speculation.

In addition, in this study, it was found that the expression of ZnT1 was upregulated in CA patients. So, we speculate that zinc concentration disturbance in patients with CA cases because of zinc transporter ZnT1. We will expand the sample size of CA patients to further explore the internal relationship and the molecular pathogenesis between HPV infection and the ZnT1 in the following studies. On this basis, the expression level of zinc in CA patients will also be our next research direction.

Summary, ZNT1 can be used as an auxiliary method for HPV detection, play a certain complementary role in HPV susceptibility and molecular mechanism, and provide a new idea for the molecular mechanism and treatment of HPV infection.

\section{References}

1. Wiraguna A, Andriani PI, Adiguna MS. Comparison of plasma zinc levels among HIV+ and HIV- subjects infected with Condyloma acuminata. Asian Pac J Cancer Prev 2019;20:943-9.

2. Yin G, Li X, Wang X, Zhang H. Effect of 5-aminolevulinic acid photodynamic therapy on the expression of apoptosis inhibitors Bcl-2 and survivin in keratinocytes of condyloma acuminatum. Photodiagnosis Photodyn Ther 2019;28:53-7.

3. Paver EC, Currie AM, Gupta R, Dahlstrom JE. Human papilloma virus related squamous cell carcinomas of the head and neck: diagnosis, clinical implications and detection of HPV. Pathology 2020;52:179-91.

4. Nadal LR, Saad SS, Lopes Filho GJ, Joaquim HPG, Manzione TDS, Manzione CR, et al. Comparison between anal cytology, high-resolution anoscopy and HPV DNA genotyping by polymerase chain reaction in the post-treatment follow-up of condylomata acuminata. Rev Col Bras Cir 2020;47: e20202543.

5. Drabina J, Huras H, Basta T, Posadzka E, Hosiawa W, Jablonski M, et al. [Prevalence of HPV DNA among male sexual partners of women diagnosed with CIN and early invasive cervical cancer].[Article in Polish]. Przegl Lek 2015;72:611-5.

6. Hammer A, de Koning MN, Blaakaer J, Steiniche T, Doorbar $\mathrm{J}$, Griffin $\mathrm{H}$, et al. Whole tissue cervical mapping of HPV infection: Molecular evidence for focal latent HPV infection in humans. Papillomavirus Res 2019;7:82-7.

7. Le Poole C, Denman CJ, Arbiser JL. Immunosuppression may be present within condyloma acuminata. J Am Acad Dermatol 2008;59:967-74.

8. Magallon J, Chiem K, Tran T, Ramirez MS, Jimenez V, Tolmasky ME. Restoration of susceptibility to amikacin by 8hydroxyquinoline analogs complexed to zinc. PLoS One 2019; 14:e0217602.

9. Delacoux E, Evstigneeff T, Leclercq M, Rettori MC, Delons S, Naret C, et al. Skin disorders and vitamin A metabolism disturbances in chronic dialysis patients: the role of zinc, retinolbinding protein, retinol and retinoic acid. Clin Chim Acta 1984;137:283-9.

10. Dhaliwal S, Nguyen M, Vaughn AR, Notay M, Chambers CJ, Sivamani RK. Effects of zinc supplementation on inflammatory skin diseases: A systematic review of the clinical evidence. Am J Clin Dermatol 2020;21:21-39.

11. Lazarczyk M, Cassonnet P, Pons C, Jacob Y, Favre M. The EVER proteins as a natural barrier against papillomaviruses: a new insight into the pathogenesis of human papillomavirus 
infections. Microbiol Mol Biol Rev 2009;73:348-70.

12. Wang X, Zhao Y, Zou X, Wang L. Relationship of P16 and $\mathrm{Ki} 67$ in recurrence of HPV infection and cervical intraepithelial neoplasia. Int J Clin Exp Pathol 2020;13:3174-80.

13. Zito Marino F, Sabetta R, Pagliuca F, Brunelli M, Aquino G, Personà $\mathrm{S}$, et al. Discrepancy of p16 immunohistochemical expression and HPV RNA in penile cancer. A multiplex in situ hybridization/ immunohistochemistry approach study. Infect Agent Cancer 2021;16:22.

14. Skoczynski M, Gozdzicka-Jozefiak A, Kwasniewska A. Cooccurrence of human papillomavirus (HPV) in newborns and their parents. BMC Infect Dis 2019;19:930.

15. Sichero L, Picconi MA, Villa LL. The contribution of Latin American research to HPV epidemiology and natural history knowledge. Braz J Med Biol Res 2020;53:e9560.

16. Farzaneh F, Nadji SA, Khosravi D, Hosseini MS, Hashemi Bahremani M, Chehrazi M, et al. Lack of HPV in benign and malignant epithelial ovarian tumors in Iran. Asian Pac J Cancer Prev 2017;18:1233-6.

17. Kazlouskaya M, Fiadorchanka N. Regression of giant condyloma acuminata after one dose of 9-valent human papillomavirus (HPV) vaccine. Int J Dermatol 2019;58:e245-e7.

18. Hinten F, Hilbrands LB, Meeuwis KAP, IntHout J, Quint WGV, Hoitsma AJ, et al. Reactivation of latent HPV infections after renal transplantation. Am J Transplant 2017;17:1563-73.

19. Al-Eitan LN, Tarkhan AH, Alghamdi MA, Al-Qarqaz FA, AlKofahi HS. Transcriptome analysis of HPV-induced warts and healthy skin in humans. BMC Med Genomics 2020;13:35.

20. Infante-Cossio P, Gonzalo DH, Hernandez-Gutierrez J, Borrero-Martin JJ. Oral inverted ductal papilloma associated with condyloma acuminata and HPV in an HIV+ patient. Int $\mathrm{J}$
Oral Maxillofac Surg 2008;37:1159-61.

21. Hultin E, Arroyo Muhr LS, Lagheden C, Dillner J. HPV transcription in skin tumors. PLoS One 2019;14:e0217942.

22. Tommasino M. HPV and skin carcinogenesis. Papillomavirus Res 2019;7:129-31.

23. Gross G, Ikenberg H, Petry KU, Pfister H, Schneede P, Schofer $\mathrm{H}$, et al. [Condyloma acuminata and other HPV-associated diseases of the genitals, anus and urethra].[Article in German]. Hautarzt 2007;58:179-86.

24. Bushara O, Miller D, Giubellino A, Schomaker M, Thyagarajan B, Nelson AC. Diagnosis of epidermodysplasia verruciformis: Two cases highlighting the role of direct HPV L1 gene sequencing. J Cutan Pathol 2019;46:436-41.

25. Pohthipornthawat N, Feldman S, Granter SR, Laga AC, Crum CP, Herfs M. Epidermodysplasia Verruciformis-like HPV Infection of the Vulva in Immunosuppressed Women. Int $\mathrm{J}$ Gynecol Pathol 2018;37:233-8.

26. Patel T, Morrison LK, Rady P, Tyring S. Epidermodysplasia verruciformis and susceptibility to HPV. Dis Markers 2010;29:199-206.

27. Arnold AW, Hofbauer GF. Human papillomavirus and squamous cell cancer of the skin-epidermodysplasia verruciformis-associated human papillomavirus revisited. Curr Probl Dermatol 2012;43:49-56.

28. Lam YW, Trinkle-Mulcahy L, Lamond AI. The nucleolus. J Cell Sci 2005;118:1335-7.

29. Eom SJ, Kim EY, Lee JE, Kang HJ, Shim J, Kim SU, et al. $\mathrm{Zn}(2+)$ induces stimulation of the c-Jun N-terminal kinase signaling pathway through phosphoinositide 3-kinase. Mol Pharmacol 2001;59:981-6.

Received for publication: 14 February 2021. Accepted for publication: 13 April 2021.

This work is licensed under a Creative Commons Attribution-NonCommercial 4.0 International License (CC BY-NC 4.0).

(C) Copyright: the Author(s), 2021

Licensee PAGEPress, Italy

European Journal of Histochemistry 2021; 65:3228

doi:10.4081/ejh.2021.3228 